\title{
Sinogenic Subdural Empyema in a Ten-Year-Old Boy with Sickle Cell Anemia
}

\author{
Ulf Nestler $^{1,2^{*}}$, Daniel Memia-Zolo ${ }^{1}$, Nidal Salloum ${ }^{1}$, Mehdi Mejdoubi ${ }^{3}$, François Lengelle ${ }^{4}$, \\ Raoul Santiago $^{5}$, William Cécile ${ }^{5}$, Remus Stegaru ${ }^{6}$, Norbert Manzo ${ }^{1}$ \\ ${ }^{1}$ Department of Neurosurgery, Centre Hospitalier Universitaire of Fort de France, Fort-de-France, French West Indies \\ ${ }^{2}$ Department of Neurosurgery, University Clinic Leipzig, Leipzig, Germany \\ ${ }^{3}$ Department of Radiology, Centre Hospitalier Universitaire of Fort de France, Fort-de-France, French West Indies \\ ${ }^{4}$ Department of Anesthesiology, Centre Hospitalier Universitaire of Fort de France, Fort-de-France, French West Indies \\ ${ }^{5}$ Department of Pediatrics, Centre Hospitalier Universitaire of Fort de France, Fort-de-France, French West Indies \\ ${ }^{6}$ Department of Oto-Rhino-Laryngology, Centre Hospitalier Universitaire of Fort de France, \\ Fort-de-France, French West Indies \\ Email: *ulf.nestler@medizin.uni-leipzig.de
}

Received July 4, 2013; revised August 4, 2013; accepted August 11, 2013

Copyright (C) 2013 Ulf Nestler et al. This is an open access article distributed under the Creative Commons Attribution License, which permits unrestricted use, distribution, and reproduction in any medium, provided the original work is properly cited.

\begin{abstract}
Objective: Sinogenic subdural empyema remains a neurosurgical emergency, even in the antibiotic era. Sickle cell disease is one of the most frequent hereditary diseases, with an incidence of around 4 in 1000 newborns, which necessitates special considerations before a neurosurgical intervention. Case Description: The case of a 10-year-old boy with sickle cell disease type HbSC is reported, who presented with meningitis about ten days after a nasal sinusitis. CT and MRI showed subdural empyema and immediate intravenous antibiotic treatment was started. The patient received partial exchange transfusion. Craniotomy allowed evacuation of empyema and repairment of a frontobasal dural defect. Endoscopic evacuation of the paranasal sinuses was done in the same anesthesia. After initial good recovery, the patient developed right sided paresis with epileptic seizures a few days later. CT scan revealed three distant empyema recurrencies which were evacuated in a second neurosurgical intervention. The child recovered without neurologic deficit, and control MRI examinations three and twelve months after surgery found frontal and parietal dural thickening as sequelae. Conclusion: Urgent neurosurgical interventions in the presence of sickle cell anemia require special anesthesiologic preparations. An immediate, multidisciplinary approach can lead to a good outcome in these life-threatening situations.
\end{abstract}

Keywords: Nasal Sinusitis; Sickle Cell Anemia; Streptococcus Pneumoniae; Subdural Empyema

\section{Introduction}

Sinogenic subdural empyema in pediatric patients is a rare entity with a reported incidence of less than 2 patients a year in many reference centres [1-4]. Even with early onset of broad-spectrum, intravenous antibiotic treatment and immediate surgical interventions it remains a life threatening disease, the mortality rate reaching up to $20 \%$ in some series [5]. Established predisposing factors comprise afro-american origin, male gender and age about 12 years [1,3]. A seasonal higher incidence with winter weather has been observed, and pathophysiological hypotheses deal with narrow anatomy and increased growth rate of the paranasal cavities in early adolescence of afro-american boys [3,6].

\footnotetext{
"Corresponding author.
}

Sickle cell anemia is the most common inherited autosomal recessive disease, with an increased frequency in afro-american populations. On the island of Martinique, the incidence is about 4 in 1000 newborns and according to the hemoglobin mutation, homocygote HbSS (62.5\%) can be distinguished from heterozygote compound forms such as HbSC (32.95\%) or HbSbeta thalassemia (4.65\%) [7]. The altered structure of hemoglobin molecules has a direct impact on erythrocytes, with reduced flexibility, sickle-shaped distortion and increased fragility [8]. Symptoms arise from capillary occlusion by distorted red blood cells and from hemolytic anemia caused by chronic intravascular hemolysis and splenic sequestration of pathologic erythrocytes. Acute syndromes can be triggered by hypoxia, hypothermia, dehydration or infection and comprise painful crises such as the acute chest syn- 
drome [9]. Among the long-term complications of sickle cell disease figure pulmonary hypertension, nephropathy, cerebral hypoperfusion syndromes and increased susceptibility to infections because of functional asplenia.

The coincidence of subdural empyema with sickle cell anemia has only been reported once in a 29-year-old male patient with fatal outcome [10]. A different case description reports the association of sickle cell anemia with brain abscess in a 29-year-old African woman, resulting in full recovery [11]. We present the case of a ten-year-old boy who suffered from a sinogenic subdural empyema in combination with HbSC compound sickle cell disease.

\section{Case Report}

The 10-year-old boy presented to the emergency unit of a community hospital with headache and fever which had been present since several days. About ten days ago, he had had discharge of nasal secretions that stopped spontaneously. At admission he was drowsy and reacted slowly, he was fully oriented and neither cranial nor peripheral neurological deficits were detected.

The HbSC subtype of sickle cell anemia had been diagnosed at birth, but due to difficult social circumstances, oral antibiotic prophylaxis had only been performed irregularly, the vaccinations were not reported and the specific follow-up examinations had not taken place. Five years ago, an episode of vaso-occlusive crisis at the elbow had been treated during a hospital stay.

Serologic work-up revealed major inflammatory signs with a white blood cell count of $22.6 \mathrm{G} / \mathrm{l}$ and a C-reactive protein value of $310 \mathrm{mg} / \mathrm{l}$. Under suspicion of meningitis lumbar puncture was performed which showed $380 / \mathrm{mm}^{3}$ white blood cells, glycemia of $3.9 \mathrm{mmol} / \mathrm{l}$ and $0.5 \mathrm{~g} / \mathrm{l}$ proteins.

CT scan and MRI revealed a left frontal, hypodense subdural lesion, with few mass effect (Figure 1). Medial to the left orbital roof, air inclusion in the subdural space suggested the presence of a dural tear with ascending sinogenic infection. The left paranasal sinuses were obliterated. Suspecting subdural empyema, a triple antibiotic therapy with cefotaxim, metronidazole and fosfomycin was started immediately and the patient was transferred to the pediatric department for the neurosurgical intervention.

At admission, the patient was clinically and neurologically stable. The hemoglobin count was $10.6 \mathrm{~g} / \mathrm{dl}$, but hemoglobin electrophoresis found $46.5 \%$ pathologic subtypes (HbS 23.8\% and $\mathrm{HbC} 22.7 \%$ ). The case was discussed with the neurosurgeon, the ENT surgeon, the anesthesiologist and the pediatrician. Finally, an immediate partial exchange transfusion was started, and after lowering the percentage of pathologic hemoglobin below $30 \%$ (HbS 13.3\%, HbC 12.4\%) the patient was transferred to the operating room.

Bifrontal craniotomy allowed the preparation of the frontal dura mater. On the right side, no defect was found, whereas on the left side a large dural tear with copious putride secretion from the subdural space was detected. Because of the vulnerability of the dural tissue direct suturing was unsuccessful. The dura was opened and the subdural spaces cleared from membranes of infectious

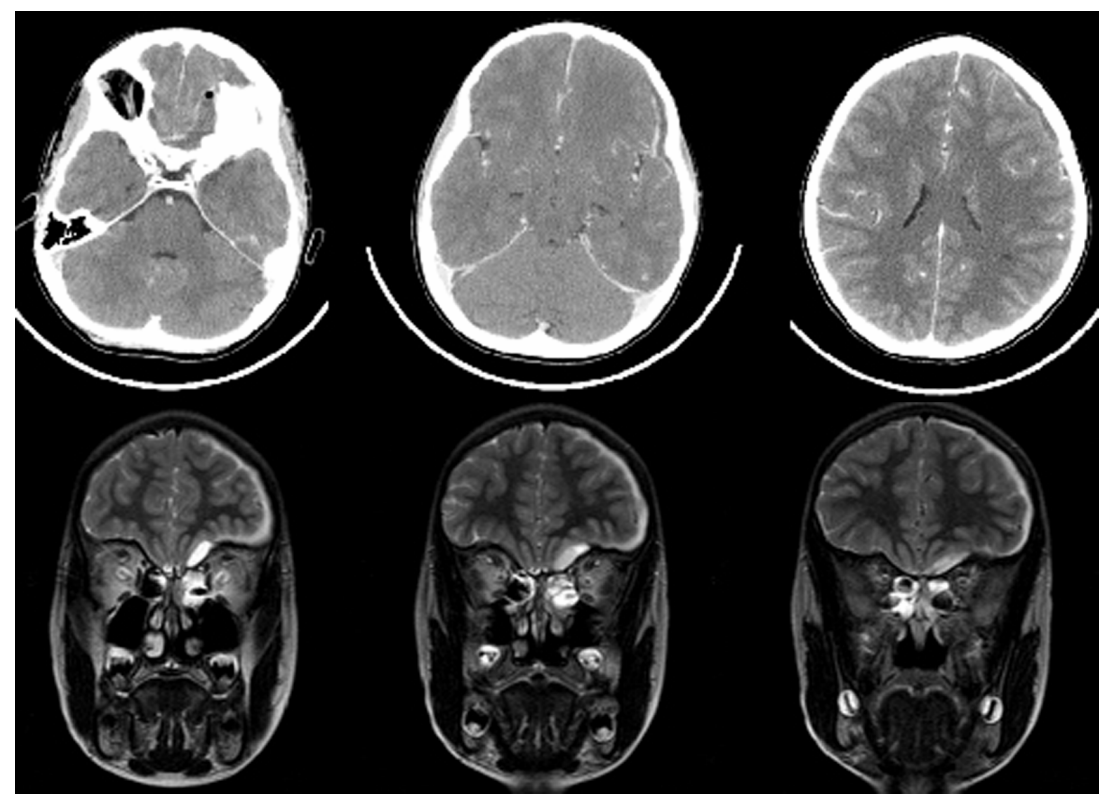

Figure 1. Upper row: contrast enhanced axial CT scan before the first operation shows left sided air inclusion within the empyema and laminar subdural spread. Lower row: coronal T2 weighted MRI depicts the extension of left subdural empyema and fluid retention in the paranasal sinuses. 
tissue and liquid pus, the arachnoid of the frontal lobe being partially destroyed by the infectious tissue. Underneath the dural tear a small notch in the cranial base was detected with adhesion of the dura mater. The tissue was coagulated and cut, leaving the bone of the cranial base without adhesions. A vascularised periosteal flap was then inserted intradurally below the frontal lobe. After watertight suture of the dura mater, the bone flap was reinserted and fixed with titanium clamps.

Immediately after the neurosurgical intervention, transnasal endoscopic surgery of the paranasal sinuses was performed which resulted in evacuation of large amounts of putrid secretions and confirmed the absence of CSF leakage.

The boy recovered well from the surgical interventions, with small, isocoric pupils and a weakness of the right levator palpebrae muscle. He complained from headache and pain in the right leg. He showed an important algophobic reaction to every kind of puncture attempt, even to non-invasive nursing procedures. The postoperative cranial CT scan revealed small residues of subdural membranes in the region of the left frontal lobe, together with a good evacuation of the supraorbital and intranasal parts of empyema. The microbiological work-up revealed Streptococcus pneumoniae, and according to the antibiogram, antibiotic treatment was continued with replacement of fosfomycin by rifampicine on the fourth postoperative day. The boy received another partial exchange transfusion in order to stabilize the hemoglobin count.

The following days, the patient developed a progressing right sided weakness, beginning as facial paralysis, then extending to the arm and the leg. Serologic controls revealed an increasing CRP value of $236 \mathrm{mg} / \mathrm{l}$ after a drop to $185 \mathrm{mg} / \mathrm{l}$ postoperatively. On the fifth postoperative day the child presented secondary generalized epileptic seizures beginning from the right side, which responded well to intrarectal admission of diazepam. A new CT scan demonstrated increasing size of three distant subdural collections on the left side, parietal, frontal and in the sylvian fissure, still with good evacuation of the former subdural empyema and free paranasal sinuses (Figure 2). The patient was immediately transferred to the operating room for a second neurosurgical intervention.

The former bifrontal suture was opened on the left side to the midline. The incision was then performed backwards and curved occipitally behind the left ear. Beginning from the former frontal bone flap, a second huge parieto-temporal flap was prepared. Upon opening of the dura mater, three subdural empyemas became visible with yellow to green appearance of the fluid and abundant membranous tissue, partly adherent to and covering the dural surface. Parietally, a tiny spot of pus was found beneath the unharmed arachnoid. In the frontal and perisylvian subdural space copious putride material was evacuated. After extensive rinsing of the intact cerebral surface, the dura was sutured and the bone flap put back in place with titanium clamps.

The boy recovered well from the second operation. The hemiplegia disappeared, the weakness of the right eye opener improved. The microbiological culture of the specimen from the second intervention remained sterile. Intravenous antibiotic therapy was complicated by a peripheral catheter infection, but could be reduced on day 20 to cefotaxim and rifampicin. Under treatment with valproic acid the patient did not suffer another seizure. A vaccination with capsular polysaccharides against pneumococcal infections was performed on day 14, after the CRP value had come down to $48 \mathrm{mg} / \mathrm{l}$.

Brain MRI on day 17 after the first operation showed no subdural or intranasal collections and revealed signs of dural thickening beneath the former trepanation sites. Transnasal control endoscopy confirmed the absence of nasal empyema. Magnetic resonance imaging was repeated on day 38, three months and one year after the first operation without reappearance of subdural or intracerebral signs of infection (Figure 3).

On day 36 after the operation the intravenous antibiotic therapy was changed to orally administered amoxicillin and rifampicin for another five weeks. The young patient could return home six weeks after his admission. At four months after his first operation, he had returned to school and did not show any neurologic deficit. A follow-up in the sickle cell outpatient clinic has been started and the boy has been included in a regular exchange transfusion program.

\section{Discussion}

Concerning subdural empyema, this case description presents several typical features of the disease, such as afro-american origin of the boy, streptococcal sinusitis and a delay of about ten days to develop signs and symptoms of meningitis. Treatment consists in immediate intravenous combination antibiotherapy, surgical closure of the entry site and evacuation of intracranial as well as intranasal putride secretions. The observed complications, such as epileptic seizures and a second neurosurgical intervention for distant empyema recurrence, are not uncommon and-as in our case-most patients recover well $[1,12]$.

The immediate start of antibiotic therapy is a crucial point in the treatment of sinogenic subdural empyema, since the intracranial infection develops from the sinusitis and precedes the clinical signs for several days. The first-hand therapy has to be adjusted after obtaining the results of the antibiogram [1].

However, this antibiotic pretreatment can lead to false 


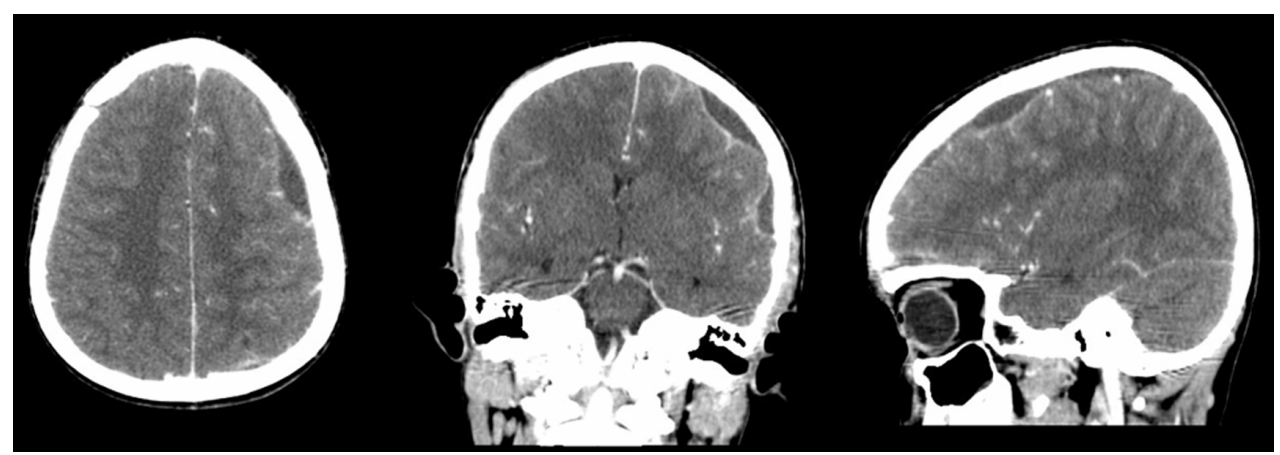

Figure 2. Contrast enhanced CT scan before the second intervention in axial, coronal and sagittal reconstruction. The three subdural empyema collections are in the left frontal region, the left sylvian fissure and in the left parietal region, separated by cortical tissue.

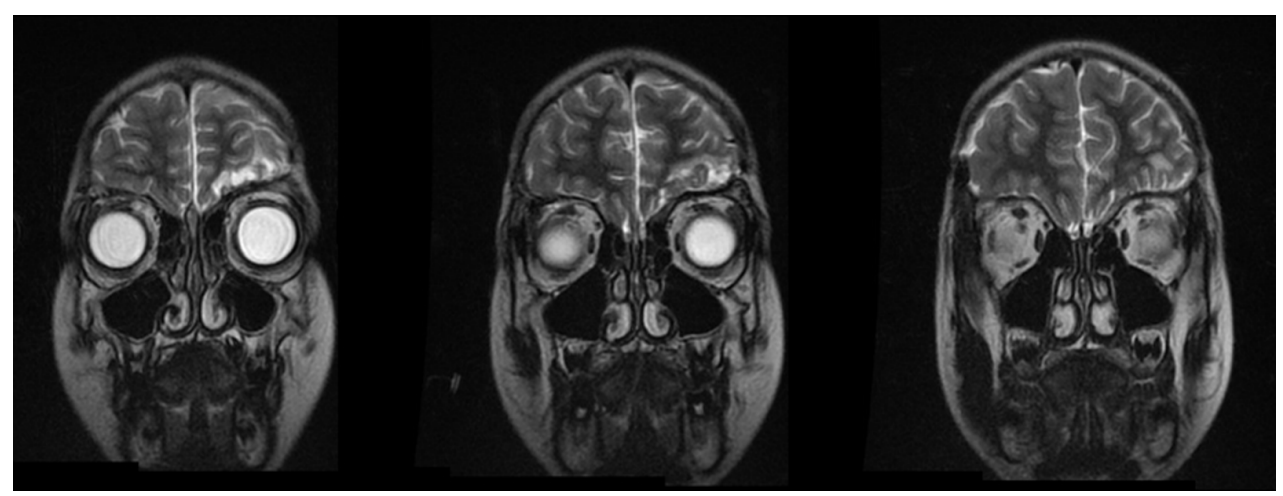

Figure 3. Coronal T2 weighted MRI three months after the first operation finding left frontal post-infectious and post-surgical alterations without subdural collections and confirming free paranasal sinuses.

negative microbiological cultures, especially in ascending infections with multiple germs, which have been observed in up to 9 percent of cases $[1,6]$. In these special situations, in case of persisting septic signs and negative bacterial cultures, alternative microbiological techniques such as PCR can help to detect and control the pathogenic organism [13].

This also underlines the importance for continuing combination antibiotic therapy for several weeks, even after normalisation of infectious parameters. Recommendations vary between 4 and 8 weeks and in our case we opted for 5 weeks of intravenous treatment followed by 5 weeks of oral antibiotics $[1,6,11]$. Up to date, there is no consensus on the duration of the antibiotic treatment after surgical intervention. A pragmatic approach can be to switch intravenous therapy to oral antibiotics as soon as the CRP value is normalized [14]. Most importantly, the empirical choice of antibiotics has to take into account the specific local prevalence of pathogens and should then be adjusted according to the antibiogram if the germ is detectable.

To the best of our knowledge the coincidence of subdural empyema with sickle cell anemia has only been reported once in an adult drug-addicted patient [10]. Anemia as an associated severe condition has been docu- mented in the large patient series, but the information does not allow to distinguish between infection-induced anemia or beforestanding hemoglobinopathies [12]. Hematologic problems are of prognostic importance and have been associated to a mortality of over $40 \%$ in these cases [6].

About one third of sickle cell patients in the French West Indies carry the HbSC aberration [7]. Given the incidence of 4 in 1000 newborn children, the probability is as low as $0.13 \%$ for a child with sinogenic subdural empyema to suffer from HbSC sickle cell disease. In so far, the case presented here has to be considered a rare occurrence. On one hand, a mere statistical coincidence could be present, because the patient group at risk for sinogenic subdural empyema has also an increased prevalence of sickle cell anemia. On the other hand, the compromise of reticulo-endothelial system immunity in sickle cell patients with increased susceptibility to pneumococcal infections might be a risk factor for subdural spread [11,15].

The use of preoperative exchange transfusions for sickle cell patients is still discussed controversely and standard recommendations are not available [16]. The benefit of an improved oxygene transport capacity has to be weighed against the risk of transfusion complications 
such as alloimmunization and hyperhemolysis [17]. In our case, in view of the stable neurological condition of the young patient before the first intervention, we opted for a partial transfusion protocol with the aim of stabilizing the hemoglobin value over $10 \mathrm{~g} / \mathrm{l}$ and reducing the percentage of pathologic HbSC below 30\% [18]. No transfusion complications were observed and the repeated prophylactic correction of parameters allowed the second operation to be performed under emergency conditions without further delay after the onset of seizures.

Sickle cell disease remains an often forgotten or even neglected entity, which needs early onset of a lifelong treatment with close follow-up of the patients in a specialized centre. The neonatal screening programs help in raising the awareness of medical caregivers and families for the patients and thus may alleviate their burden [7, $18]$.

\section{Conclusion}

Coincidence of sinogenic subdural empyema with sickle cell anemia is rare, but potentially life-threatening combination of diseases. An immediate, multidisciplinary approach allows for rapid treatment with early detection of complications and can result in a favourable outcome for the young patients.

\section{REFERENCES}

[1] S. Gupta, S. Vachhrajani, A. Kulkarni, M. D. Taylor, P. Dirks, J. M. Drake and J. T. Rutka, "Neurosurgical Management of Extraaxial Central Nervous System Infections in Children,” Journal of Neurosurgery: Pediatrics, Vol. 7, No. 5, 2011, pp. 441-451.

[2] C. W. Hicks, J. G. Weber, J. R. Reid and M. Moodley, "Identifying and Managing Intracranial Complications of Sinusitis in Children," Pediatric Infectious Disease Journal, Vol. 30, No. 3, 2011, pp. 222-226.

http://dx.doi.org/10.1097/INF.0b013e3181f86398

[3] J. H. Piatt, "Intracranial Suppuration Complicating Sinusitis among Children: An Epidemiological and Clinical Study," Journal of Neurosurgery: Pediatrics, Vol. 7, No. 6, 2011, pp. 567-574. http://dx.doi.org/10.3171/2011.3.PEDS10504

[4] T. P. Tsou, P. I. Lee, C. Y. Lu, L. Y. Chang, L. M. Huang, J. M. Chen, P. R. Hsueh and C. Y. Lee, "Microbiology and Epidemiology of Brain Abscess and Subdural Empyema in a Medical Center: A 10-Year Experience,” Journal of Microbiology, Immunology and Infection, Vol. 42, No. 5, 2009, pp. 405-412.

[5] S. C. Leong, L. K. Waugh, A. Sinha and S. De, "Clinical Outcomes of Sinogenic Intracranial Suppuration: The Alder Hey Experience,” Ann Otol Rhinol Laryngol, Vol. 120, No. 5, 2011, pp. 320-325.

[6] N. Nathoo, S. S. Nadvi, J. R. van Dellen and E. Gouws, "Intracranial Subdural Empyemas in the Era of Computed Tomography: A Review of 699 Cases,” Neurosurgery,
Vol. 44, No. 3, 1999, pp. 529-536. http://dx.doi.org/10.1097/00006123-199903000-00055

[7] F. M. Mbou, L. Martineau, A. Eischen, G. Elana and E. Dupuis, "Clinical Course (Ten Years) of Sickle Cell Disease in Martinique after Neonatal Screening," Archives de Pédiatrie, Vol. 11, No. 1, 2004, pp. 57-58.

http://dx.doi.org/10.1016/j.arcped.2003.09.050

[8] A. K. Ould Amar, O. Delattre, C. Godbille, O. Béra, P. A. Halbout and Y. Catonné, "Assessment of the Use of Transfusion Therapy and Complications in Orthopedic Surgery in Patients with Sickle-Cell Anemia: Retrospective Study," Transfusion Clinique et Biologique, Vol. 10, No. 2, 2003, pp. 61-66.

http://dx.doi.org/10.1016/S1246-7820(03)00021-1

[9] Z. M. Al-Samak, M. M. Al-Falaki and A. A. Pasha, “Assessment of Perioperative Transfusion Therapy and Complications in Sickle Cell Disease Patients Undergoing Surgery,” Middle East Journal of Anesthesiology, Vol. 19, No. 5, 2008, pp. 983-996.

[10] T. Moon, R. Y. Lin and A. F. Jahn, "Fatal Frontal Sinusitis Due to Neisseria sicca and Eubacterium lentum," Journal of Otolaryngology, Vol. 15, No. 3, 1986, pp. 193195.

[11] S. Kuruvath, S. Basu, J. P. Elwitigala, A. Yaneza, S. S. Namnyak and A. R. Aspoas, "Salmonella Enteritidis Brain Abscess in a Sickle Cell Disease Patient: Case Report and Review of the Literature,” International Journal of Infectious Diseases, Vol. 12, No. 3, 2008, pp. 298-302. http://dx.doi.org/10.1016/j.ijid.2007.02.004

[12] V. S. Madhugiri, B. V. Sastri, U. Srikantha, A. D. Banerjee, S. Somanna, B. I. Devi, B. A. Chandramouli and P. Pandey, "Focal Intradural Brain Infections in Children: An Analysis of Management and Outcome,” Pediatric Neurosurgery, Vol. 47, No. 2, 2011, pp. 113-124. http://dx.doi.org/10.1159/000330542

[13] R. Bhatia, K. Harris, J. Hartley, O. Jeelani and W. Harkness, "Serial PCR Genetic Load Determination in the Surgical Management of Pneumococcal Intracranial Sepsis," Child's Nervous System, Vol. 28, No. 4, 2012, pp. 515520. http://dx.doi.org/10.1007/s00381-012-1715-y

[14] T. S. Cole, M. E. Clark, A. J. Jenkins and J. E. Clark, "Pediatric Focal Intracranial Suppuration: A UK SingleCenter Experience,” Child's Nervous System, Vol. 28, No. 12, 2012, pp. 2109-2114. http://dx.doi.org/10.1007/s00381-012-1877-7

[15] T. V. Adamkiewicz, S. Sarnaik, G. R. Buchanan, R. V. Iyer, S. T. Miller, C. H. Pegelow, Z. R. Rogers, E. Vichinsky, J. Elliott, R. R. Facklam, K. L. O’Brien, B. Schwartz, C. A. Van Beneden, M. J. Cannon, J. R. Eckman, H. Keyserling, K. Sullivan, W. Y. Wong and W. C. Wang, "Invasive Pneumococcal Infections in Children with Sickle Cell Disease in the Era of Penicillin Prophylaxis, Antibiotic Resistance, and 23-Valent Pneumococcal Polysaccharide Vaccination,” Journal of Pediatrics, Vol. 143, No. 4, 2003, pp. 438-444. http://dx.doi.org/10.1067/S0022-3476(03)00331-7

[16] P. G. Firth, K. N. McMillan, C. M. Haberkern, M. Yaster, M. A. Bender and S. R. Goodwin, "A Survey of Perioperative Management of Sickle Cell Disease in North 
America,” Pediatric Anesthesia, Vol. 21, No. 1, 2011, pp. 43-49.

http://dx.doi.org/10.1111/j.1460-9592.2010.03415.x

[17] L. Fisher, "Perioperative Care of the Patient with Sickle Cell Disease,” AORN Journal, Vol. 93, No. 1, 2011, pp.
150-156. http://dx.doi.org/10.1016/j.aorn.2010.08.019

[18] D. C. Rees, T. N. Williams and M. T. Gladwin, "SickleCell Disease,” Lancet, Vol. 376, No. 9757, 2010, pp. 2018-2031.

http://dx.doi.org/10.1016/S0140-6736(10)61029-X 